\title{
Depressão durante a gestação: um estudo sobre a associação entre fatores de risco e de apoio social entre gestantes
}

\section{Depression during pregnancy: a study about the association between risk factors and social support among pregnant}

\author{
Daianna Lima Thiengoํ, Jacqueline Fernandes de Cintra Santos², Diego Lima Fonseca', \\ Lúcia Abelha², Giovanni Marcos Lovisi²
}

\begin{abstract}
Resumo
A ausência de apoio social e outros fatores podem tornar gestantes mais suscetíveis à ocorrência de transtornos mentais como a depressão. O objetivo deste estudo foi investigar a associação entre apoio social percebido e prevalência de depressão em gestantes atendidas em uma unidade básica de saúde de Nova Iguaçu, Rio de Janeiro. Participaram dele 100 gestantes, das quais foram verificadas as características sociodemográficas, condições médicas/obstétricas, eventos estressantes e apoio social. A depressão foi avaliada pela Composite International Diagnostic Interview. A prevalência da depressão na gravidez foi 18\% (IC95\% 12,2-23,8), sendo os fatores associados na análise final: estar solteira, desempregada, possuir história anterior de depressão e tabagismo. Houve relação entre a falta de apoio social e a depressão na análise inicial $(p<0,05)$. A ausência de apoio de informação e interação durante o período pré-natal, e sua associação inicial com a depressão, evidencia a fragilidade do serviço de saúde no atendimento integral às gestantes. Nossos resultados demonstram que intervenções psicossociais e políticas sociais deveriam ser implementadas para esta população.
\end{abstract}

Palavras-chave: gravidez; apoio social; fatores de risco; depressão.

\begin{abstract}
Lack of social support and other factors can cause pregnant more susceptible to mental disorders such as depression. The aim of this study was to investigate the association between perceived social support and prevalence of depression in pregnant attended in a primary care unit of Nova Iguaçu, Rio de Janeiro. The study included 100 women and verified the sociodemographic characteristics, obstetric and medical complications, stressful life events and social support. Depression was evaluated using the Composite International Diagnostic Interview. The prevalence of depression during pregnancy was $18 \%(95 \% \mathrm{Cl} 12.2-23.8)$, and the associated factors in the final analysis were: being single, unemployed, with a prior history of depression and smoking. In the initial analysis, there was significant association $(p<0.05)$ between lack of social support and depression. The absence of information and social interaction during the prenatal period, and its initial association with depression, shows the failings of the full health care services for pregnant. Our findings show that psychosocial interactions and social policies should be implemented for this population.
\end{abstract}

Keywords: pregnancy; social support; risk factors; depression.

Trabalho realizado no Centro de Saúde Dr. Vasco Barcelos - Nova Iguaçu (RJ), Brasil.

1Doutoranda em Saúde Coletiva pelo Instituto de Estudos em Saúde Coletiva da Universidade Federal do Rio de Janeiro (UFRJ) - Nova Iguaçu (RJ), Brasil. 2Professor(a) Adjunto(a) do Instituto de Estudos em Saúde Coletiva da UFRJ - Nova Iguaçu (RJ), Brasil.

Endereço para correspondência: Daianna Lima Thiengo - Avenida Doutor Carvalhaes, 1048, casa - Rocha Sobrinho - CEP: 26572-530 - Mesquita (RJ), Brasil E-mail: daianna.thiengo@yahoo.com.br

Fonte de financiamento: Fundação Carlos Chagas de Amparo à Pesquisa do Estado do Rio de Janeiro (FAPERJ) e Coordenação de Aperfeiçoamento de Pessoal de Nível Superior (CAPES).

Conflito de interesse: nada a declarar. 


\section{INTRODUÇÃO}

Desde a década de 1960, estudos indicam que o apoio social pode estar associado à etiologia, à precipitação, ao curso e recuperação de indivíduos com transtornos mentais ${ }^{1-3}$.

Duas teorias explicam a associação entre apoio social e saúde mental. A primeira refere-se ao fato de que a ausência desse tipo de apoio afeta diretamente a saúde mental, desencadeando transtornos. Já a segunda diz respeito ao modelo de eventos estressores, transtornos mentais nos quais o apoio social funcionaria como mediador do estresse (efeito buffer) modificando o seu efeito, ou seja, o indivíduo sem apoio social teria mais predisposição aos transtornos mentais ${ }^{4}$.

O apoio social é um construto multidimensional que envolve o conforto, carinho, a assistência e/ou informações que alguém recebe de contatos sociais, estando ainda relacionado à percepção que a pessoa tem do seu mundo social, estratégias para estabelecer vínculos e os recursos que lhe são ofereci$\operatorname{dos}^{5-7}$. O papel do apoio social nas diversas fases da vida é fundamental para o amortecimento de eventos estressantes que ocorrem no cotidiano, principalmente em momentos em que se observam diversas modificações psicossociais e fisiológicas, como é o caso da gestação ${ }^{8-10}$.

A literatura científica indica que o período gravídico-puerperal é a fase de maior frequência de transtornos mentais na mulher, principalmente no primeiro e no terceiro trimestres de gestação e nos primeiros 30 dias do pós-parto ${ }^{11}$, sendo a depressão o transtorno mais frequente ${ }^{11,12}$.

A prevalência de depressão gestacional é de aproximadamente $15 \%$ entre países desenvolvidos (de 10 a $15 \%$ ) e cerca de $22 \%$ em países em desenvolvimento (entre 20 e $25 \%)^{13-20}$. Ela está associada a fatores como história prévia de transtorno psiquiátrico, características sociodemográficas (baixa renda), estado civil solteira ou separada, gravidez não planejada, complicações obstétricas, tabagismo, alcoolismo, eventos estressantes (furto e roubo, por exemplo) e ausência de apoio social ${ }^{12,17,21-25}$.

No Brasil, há poucos estudos sobre a depressão durante a gravidez, e grande parte deles foram desenvolvidos em ambiente hospitalar ${ }^{16}$ e com grávidas adolescentes ${ }^{20,26,27}$, o que demonstra predisposição na avaliação de gestantes de risco, desta forma, mais propensas à depressão gestacional. Outras pesquisas utilizaram amostras não representativas da população ${ }^{28}$ e escalas para rastreamento de sintomas depressivos e não para o diagnóstico de depressão ${ }^{29-31}$. Cabe ressaltar que a maioria desses estudos não utilizou instrumentos validados para avaliar os fatores de risco, em especial o apoio social ${ }^{13,26,28,29,31}$.

O maior conhecimento da associação entre apoio social e outros fatores relacionados à depressão gestacional é de fundamental importância na busca de estratégias para a implementação de políticas de assistência materno-infantil no Brasil. Deste modo, torna-se necessária maior compreensão sobre o assunto, por meio de estudos que possam preencher lacunas metodológicas como a utilização de instrumentos específicos e validados para a avaliação de depressão e de outros fatores de risco e amostras representativas, além de locais de estudo que possam atender gestantes de baixo risco, minimizando os vieses de seleção.

Neste contexto, o objetivo principal deste estudo foi investigar a relação entre apoio social percebido pela gestante e prevalência de depressão em grávidas atendidas em uma unidade básica de saúde de Nova Iguaçu, no Rio de Janeiro.

\section{MATERIAL E MÉTODOS}

\section{Local do estudo}

O presente trabalho foi realizado no Centro de Saúde Dr. Vasco Barcelos, localizado no município de Nova Iguaçu, na Baixada Fluminense, Rio de Janeiro, com característica de atenção primária.

Trata-se da unidade básica de saúde maior e mais completa do município, com atendimento ambulatorial, clientela de demanda espontânea e referenciada (Sistema Único de Saúde SUS), nos turnos da manhã e tarde, em clínicas de atendimento médico; seis clínicas especializadas; serviços de reabilitação; de enfermagem e imunização; de apoio, como farmácia e serviço social; e investigação epidemiológica. Possui equipe composta por 28 médicos, além de 139 outros profissionais. Porém, não conta com a especialidade psiquiatria.

Em relação ao Serviço de Pré-Natal, o Centro de Saúde recebe mensalmente cerca de 25 gestantes no terceiro trimestre de gravidez, sendo os atendimentos semanais, toda segunda-feira, nos turnos da manhã e tarde, com equipes formadas por dois médicos ginecologistas e cinco enfermeiras.

\section{População do estudo}

A população do estudo foi constituída por todas as gestantes no terceiro trimestre de gestação atendidas no período de março a setembro de 2011 no Centro de Saúde Dr. Vasco Barcelos.

\section{Coleta de dados}

As gestantes foram abordadas na sala de espera do serviço de pré-natal e conduzidas a uma sala separada, onde foram explicados os objetivos e as questões éticas relacionadas ao estudo. Após a consulta do pré-natal, as gestantes que consentiam participar assinavam o termo de consentimento livre e esclarecido. 
As entrevistas foram realizadas pela pesquisadora principal do projeto, com duração de cerca de 40 minutos. Durante elas, as gestantes responderam perguntas sobre sua situação sociodemográfica, história patológica pregressa, obstétrica e psiquiátrica, eventos de vida estressantes no último ano, apoio social e depressão. Foram utilizados os seguintes instrumentos:

- Avaliação da depressão: A Composite International Diagnostic Interview (CIDI; versão 2.1), desenvolvida e padronizada pela Organização Mundial de Saúde (OMS), é uma entrevista diagnóstica estruturada que pode ser utilizada por entrevistadores leigos treinados. Ela fornece diagnóstico de transtornos mentais baseado nos critérios da Classificação Internacional de Doenças (CID-10) ${ }^{32} \mathrm{e}$ Statistical Manual of Mental Disorders (DSM-IV) ${ }^{33}$ a partir de um algoritmo computadorizado. Essa ferramenta detecta os transtornos mentais dos últimos 12 meses e durante a vida.

O CIDI foi desenvolvido para uso em diferentes culturas e contextos, tendo sido validado em vários países, o que permite a comparação com estudos internacionais. Além disso, fornece diagnóstico diferencial para os sintomas típicos da gravidez, muitas vezes confundidos com sintomas depressivos. Neste estudo, a presença da depressão baseou-se nos critérios diagnósticos do CID-10 $0^{32}$. O CIDI foi traduzido do inglês para o português e validado para o uso no Brasil (kappa $=0,97$ e 0,93) $)^{34}$.

- Apoio social: Para verificar a presença ou ausência de apoio social, foi utilizada a escala Medical Outcome Studies (MOS), que mede a percepção do indivíduo sobre o grau de apoio social ${ }^{35}$. O questionário é composto por 19 itens, compreendendo 5 dimensões funcionais de apoio social: material (4 perguntas: provisão de recursos práticos); afetivo (3 perguntas: demonstrações físicas de amor e afeto); emocional (4 perguntas: expressões de compreensão e sentimentos de confiança); interação social positiva (4 perguntas: disponibilidade de pessoas para se divertirem ou relaxarem) e informação (4 perguntas: disponibilidade de pessoas para a obtenção de conselhos ou orientações). Para cada item, a gestante indicava com que frequência considerava cada tipo de apoio por uma escala ordinal: nunca (1), raramente (2), às vezes (3), quase sempre (4) e sempre (5).

Para a análise do apoio social percebido, foram calculados os escores por meio da soma dos pontos das respostas dadas às perguntas de cada uma das dimensões e divididos pelo número máximo de pontos possível de ser obtido na mesma dimensão. A fim de padronizar os resultados, tendo em vista que as dimensões eram constituídas por diferentes números de perguntas, a razão (total de pontos obtidos dividido pela pontuação máxima da dimensão) foi multiplicada por $100^{35}$. A interpretação do resultado dos escores leva em conta que quanto maior o total alcançado, maior o nível de apoio social ${ }^{36}$. Assim, o primeiro tercil é constituído pelos escores mais baixos, o segundo pelos níveis intermediários e o terceiro pelos escores mais altos.

Embora a escala de apoio social seja distribuída em três escores (baixo, intermediário e alto), optamos por analisá-la em dois níveis: ausência e presença, considerando que o primeiro foi constituído pelos níveis baixo e intermediário e o segundo pelo nível alto. Esta divisão foi realizada com o objetivo de padronizar todas as variáveis do estudo em categóricas, o que ajudou na análise dos dados. O mesmo procedimento foi realizado por outros estudos que avaliaram apoio social em gestantes e utilizaram esta mesma escala ${ }^{16,35}$.

Este instrumento foi submetido a procedimentos padronizados de tradução e adaptação ${ }^{37}$. A consistência interna estimada pelo coeficiente alpha de Cronbach entre as dimensões de apoio social variou entre 0,75 e 0,91 no teste e entre 0,86 e 0,93 no reteste, demonstrando boa confiabilidade $^{37}$. Foi recomendada a sua utilização inclusive para a população de gestantes ${ }^{38}$.

- Eventos de vida estressantes: Para identificar a presença de eventos de vida produtores de estresse no último ano, a escala utilizada foi a Stressful Life Events ${ }^{39}$, adaptada e validada para uso no Brasil ${ }^{40}$. A avaliação da confiabilidade foi considerada muito boa para quase todos os eventos (kappa $=0,61$ a 0,80$)$. Por meio dessa escala, foram investigados, neste estudo, 14 eventos estressantes: conflitos matrimoniais; história de separação ou divórcio; perda do emprego; conflitos com pessoas próximas; problema físico grave; problemas financeiros; envolvimento judicial; história de acidente de trânsito, incêndio ou catástrofe; história de sequestro; falecimento de pessoa próxima; história de abuso sexual; história de furto; história de agressão e história de assalto residencial ${ }^{40}$.

- Variáveis clínicas, obstétricas e psiquiátricas: Foi utilizado um questionário para variáveis clínicas por meio da história patológica pregressa (doenças agudas, crônicas transmissíveis e não transmissíveis); variáveis obstétricas incluindo planejamento da gravidez, número de gestações, doenças gestacionais atuais e anteriores, suas intercorrências e desfechos desfavoráveis em gestações anteriores (abortamentos, prematuridade e baixo peso ao nascer); e psiquiátrica (história anterior de depressão, tratamento psiquiátrico prévio e uso de tabaco, álcool e drogas ilícitas $\left.{ }^{16,41,42}\right)$. 
- Variáveis sociodemográficas: Procedeu-se a coleta de dados sobre idade, estado civil, escolaridade, cor, naturalidade, nacionalidade e profissão. Os critérios de classificação econômica da Associação Nacional de Empresas de Pesquisa (ANEP) foram usados na estratificação socioeconômica, baseada em informações sobre o grau de instrução do chefe da família e a posse e número de eletrodomésticos na casa. Esta classificação estratifica as entrevistadas em cinco diferentes categorias (de A a E), porém, vale ressaltar que esta variável foi agrupada em três categorias: alta (A e B), média (C) e baixa (D e E) $)^{41-43}$ a fim de facilitar a organização dos dados.

\section{Análise dos dados}

A prevalência de depressão gestacional foi calculada a partir da definição dos casos pela presença de depressão de acordo com o CID-10. Para a análise univariada dos dados foram utilizadas a frequência, a média e o desvio padrão. Já para a bivariada, calcularam-se a odds ratio (OR) e o intervalo de confiança (IC) de 95\%, o Teste do $X^{2}$, testes de Fisher e de Pearson, que mensuraram as associações entre variáveis categóricas. Em todos estes testes considerou-se nível de significância de 5\%.

As variáveis associadas significativamente com a depressão gestacional, considerando o valor $\mathrm{p} \leq 0,10$, entraram na regressão logística. Para o modelo final foram calculados odds ratio com um IC de 95\%, e o programa Statistical Package for the Social Sciences (SPSS), versão 11.0, foi utilizado para digitação e análise estatística dos dados.

\section{Aspectos éticos}

O estudo foi submetido e aprovado pelo Comitê de Ética em Pesquisa do Instituto de Estudos em Saúde Coletiva da Universidade Federal do Rio de Janeiro (UFRJ). Todas as participantes assinaram o termo de consentimento livre esclarecido antes de serem entrevistadas.

Nos casos de impedimento de assinatura, foi obtida impressão digital. Cabe ressaltar que as gestantes que apresentaram depressão foram encaminhadas às unidades de saúde que prestavam atendimento adequado.

\section{RESULTADOS}

Foram convidadas a fazer parte do estudo 104 gestantes, porém 4 se recusaram, resultando na taxa de participação de $96,1 \%$. As que se recusaram não informaram a razão. A prevalência de depressão durante a gestação neste estudo foi de $18 \%$ (IC95\% 12,2-23,8), e a prevalência de depressão por toda a vida $24 \%$ (IC95\% 18,2-29,8).

\section{Características sociodemográficas}

A idade das gestantes variou de 14 a 39 anos, com média de 25,2 anos $(\mathrm{DP}=6,176)$. A faixa etária de maior proporção foi a de 20 a 29 anos (51\%) e $26 \%$ da amostra era composta por adolescentes de 14 a 19 anos. Em relação ao estado civil, a maioria das entrevistadas era casada (54\%), apenas $2 \%$ alegaram estar divorciadas naquele momento e nenhuma afirmou viuvez. Deste modo, as duas últimas categorias não foram consideradas na análise estatística, já que a frequência foi baixa $(n=2)$.

A maior parte da amostra possuía mais de 8 anos de estudo (51\%), se declarou não branca (71\%), era natural do estado do Rio de Janeiro (88\%), desempregada (42\%) e enquadrava-se na classe socioeconômica baixa (72\%). O fato de estar solteira e desempregada foi associado significativamente à depressão durante a gestação na análise bivariada (Tabela 1).

\section{Variáveis clínicas, obstétricas e psiquiátricas}

Neste estudo, 70\% das gestantes não planejaram a atual gravidez, $40 \%$ eram primíparas, $24 \%$ relataram história anterior de depressão e 15\% afirmaram história anterior de abortamentos espontâneos. As variáveis associadas significativamente com a depressão durante a gestação na análise bivariada foram: história anterior de depressão, tabagismo e uso abusivo de álcool (Tabela 2).

\section{Eventos de vida estressantes}

Sobre este tópico, $88 \%$ das gestantes relataram ter sofrido pelo menos um tipo de evento estressante no último ano, sendo os mais comuns: conflito matrimonial (49\%), problema financeiro (38\%), falecimento de pessoa próxima (38\%), conflitos com familiares, amigos e vizinhos (36\%), história de separação ou divórcio (28\%) e perda de emprego (24\%). História de separação/divórcio foi o único evento de vida estressante associado significativamente à depressão durante a gestação (Tabela 3).

\section{Apoio social}

No que diz respeito ao apoio social, a média dos escores situou-se em torno de 80 pontos $(\mathrm{DP}=16,4)$. $\mathrm{O}$ apoio afetivo foi o que apresentou maior média: $91,79(\mathrm{DP}=14,80)$, enquanto o material teve a menor: $85,65(\mathrm{DP}=19,13)$. Cabe relembrar que esta escala possui três escores (baixo, intermediário e alto), porém optamos por analisá-la em dois níveis: ausência e presença, em que ausência foi constituída pelos níveis baixo e intermediário e presença pelo alto, a fim de padronizar todas as variáveis do estudo em categóricas ${ }^{16,35,37}$.

Foi evidenciado que a maioria das gestantes relatou ausência de apoio de informação (42\%), de material (33\%) e emocional (30\%). As ausências de apoio de informação e de interação positiva apresentaram associação significativa na análise bivariada (Tabela 4). 
Tabela 1. Características sociodemográficas e sua associação com a depressão gestacional em 100 mulheres atendidas em uma unidade básica de saúde na cidade de Nova Iguaçu (RJ), com intervalo de confiança de $95 \%$

\begin{tabular}{|c|c|c|c|c|c|c|}
\hline \multirow{2}{*}{ Características } & \multicolumn{4}{|c|}{ Depressão } & \multirow{2}{*}{ OR (IC95\%) } & \multirow{2}{*}{ Valor $\mathrm{p}$} \\
\hline & $\%$ & $\mathbf{n}$ & $\%$ & IC95\% & & \\
\hline \multicolumn{7}{|l|}{ Faixa etária (anos) } \\
\hline Adolescentes (14-19) & 26,0 & 7 & 7,0 & $4,6-18,6$ & $2,11(0,7-6,1)$ & 0,17 \\
\hline Adultas $(\geq 20)$ & 74,0 & 11 & 11,0 & $4,2-17,8$ & 1 & \\
\hline \multicolumn{7}{|l|}{ Estado civil } \\
\hline Solteira & 44,0 & 12 & 12,0 & $3,2-20,8$ & $3,86(1,2-11,8)$ & 0,01 \\
\hline Casada & 54,0 & 5 & 5,0 & $2,8-12,8$ & 1 & \\
\hline \multicolumn{7}{|c|}{ Escolaridade (anos de estudo) } \\
\hline$\leq 8$ & 49,0 & 12 & 12,0 & $3,8-20,2$ & $2,09(0,7-6,1)$ & 0,14 \\
\hline$>8$ & 51,0 & 6 & 6,0 & $2,0-8,0$ & 1 & \\
\hline \multicolumn{7}{|l|}{ Cor } \\
\hline Branca & 25,0 & 4 & 4,0 & $0,1-15,6$ & $0,65(0,1-2,1)$ & 0,48 \\
\hline Não branca & 71,0 & 14 & 14,0 & $7,2-20,8$ & 1 & \\
\hline \multicolumn{7}{|l|}{ Naturalidade } \\
\hline Rio de Janeiro & 88,0 & 16 & 16,0 & $9,7-22,2$ & $1,11(0,2-5,5)$ & 0,89 \\
\hline Outro & 12,0 & 2 & 2,0 & $0,1-19,0$ & 1 & \\
\hline \multicolumn{7}{|l|}{ Profissão } \\
\hline Desempregada & 42,0 & 11 & 11,0 & $2,0-20,1$ & $2,58(0,9-7,3)$ & 0,07 \\
\hline Trabalho formal & 21,0 & 2 & 2,0 & $0,1-14,8$ & $0,47(0,0-4,0)$ & 0,48 \\
\hline Trabalho informal & 10,0 & 1 & 1,0 & $0,1-19,3$ & $0,41(0,0-1,9)$ & 0,26 \\
\hline Outros $^{\star}$ & 27,0 & 4 & 4,0 & $0,1-15,6$ & 1 & \\
\hline \multicolumn{7}{|l|}{ Classe social } \\
\hline D e E (baixa) & 72,0 & 13 & 13,0 & $6,1-19,9$ & $1,01(0,3-3,1)$ & 0,61 \\
\hline C (média) & 15,0 & 2 & 2,0 & $0,1-17,2$ & $0,66(0,3-5,8)$ & 0,98 \\
\hline A e B (alta) & 13,0 & 3 & 3,0 & $0,1-19,1$ & 1 & \\
\hline
\end{tabular}

*Dona de casa (16\%) e estudante (11\%); n=100; IC: intervalo de confiança; OR: odds ratio

Tabela 2. Variáveis clínicas e obstétricas associadas à depressão gestacional em 100 mulheres atendidas em uma unidade básica de saúde na cidade de Nova Iguaçu (RJ), com intervalo de confiança de $95 \%$

\begin{tabular}{|c|c|c|c|c|c|c|}
\hline \multirow{2}{*}{ Variáveis clínicas } & \multicolumn{4}{|c|}{ Depressão } & \multirow{2}{*}{ OR (IC95\%) } & \multirow{2}{*}{ Valor $\mathrm{p}$} \\
\hline & $\%$ & $\mathbf{n}$ & $\%$ & IC95\% & & \\
\hline $\begin{array}{l}\text { História patológica pregressa } \\
\text { Outros }\end{array}$ & \multicolumn{5}{|c|}{ História patológica pregressa } & 0,29 \\
\hline \multicolumn{7}{|l|}{ História psiquiátrica } \\
\hline História anterior de depressão & 24,0 & 9 & 9,0 & $3,1-21,1$ & $4,46(1,5-13,1)$ & 0,00 \\
\hline Tratamento psiquiátrico anterior & 6,0 & 2 & 2,0 & $0,2-24,0$ & $2,43(0,4-14,4)$ & 0,35 \\
\hline Tabagismo & 6,0 & 4 & 4,0 & $0,1-26,0$ & $11,00(1,9-68,4)$ & 0,00 \\
\hline Uso abusivo de álcool & 5,0 & 3 & 3,0 & $0,2-26,2$ & $8,00(1,2-52,0)$ & 0,02 \\
\hline \multirow{2}{*}{ Variáveis obstétricas } & \multicolumn{4}{|c|}{ Depressão } & \multirow{2}{*}{ OR (IC95\%) } & \multirow{2}{*}{ Valor $\mathrm{p}$} \\
\hline & $\%$ & $\mathbf{n}$ & $\%$ & IC95\% & & \\
\hline Primíparas & 40,0 & 7 & 7,0 & $2,2-16,2$ & $0,94(0,3-2,6)$ & 0,91 \\
\hline História de abortamentos induzidos & 6,0 & 0 & 0,0 & - & - & - \\
\hline História de abortamentos espontâneos & 15,0 & 1 & 1,0 & $0,1-15,8$ & $0,28(0,0-0,3)$ & 0,21 \\
\hline História de doenças gestacionais & 5,0 & 1 & 1,0 & $0,3-27,0$ & $1,14(0,1-10,0)$ & 0,90 \\
\hline História de baixo peso ao nascer & 2,0 & 1 & 1,0 & $0,4-29,8$ & $4,70(0,2-79,0)$ & 0,29 \\
\hline História de prematuridade & 3,0 & 1 & 1,0 & $0,3-33,1$ & $2,35(0,2-27,0)$ & 0,51 \\
\hline Atual gravidez não planejada & 70,0 & 4 & 4,0 & $2,8-10,8$ & $0,61(0,1-2,1)$ & 0,42 \\
\hline
\end{tabular}

$\mathrm{n}=100$; IC: intervalo de confiança; OR: odds ratio 
Tabela 3. Eventos de vida estressantes associados à depressão gestacional em 100 mulheres atendidas em uma unidade básica de saúde na cidade de Nova Iguaçu (RJ), com intervalo de confiança de $95 \%$

\begin{tabular}{|c|c|c|c|c|c|c|}
\hline \multirow{2}{*}{ Eventos de vida estressantes } & \multicolumn{4}{|c|}{ Depressão } & \multirow{2}{*}{ OR (IC95\%) } & \multirow{2}{*}{ Valor $p$} \\
\hline & $\%$ & n & $\%$ & IC95\% & & \\
\hline Conflitos matrimoniais & 49,0 & 9 & 9,0 & $0,8-17,2$ & $1,05(0,3-2,9)$ & 0,92 \\
\hline História de separação/divórcio & 28,0 & 8 & 8,0 & $3,2-19,0$ & $2,48(0,8-7,1)$ & 0,09 \\
\hline Perda do emprego & 24,0 & 5 & 5,0 & $3,1-17,0$ & $1,27(0,4-4,0)$ & 0,68 \\
\hline Conflitos com pessoas próximas & 36,0 & 9 & 9,0 & $0,6-18,6$ & $2,03(0,7-5,7)$ & 0,17 \\
\hline Problema físico grave & 7,0 & 2 & 2,0 & $0,2-22,1$ & $1,92(0,3-10,0)$ & 0,47 \\
\hline Problemas financeiros & 38,0 & 6 & 6,0 & $3,4-15,4$ & $0,78(0,2-2,2)$ & 0,65 \\
\hline Envolvimento judicial & 11,0 & 2 & 2,0 & $0,1-19,4$ & $1,01(0,1-5,1)$ & 0,98 \\
\hline História de acidente, incêndio ou catástrofe & 5,0 & 1 & 1,0 & $0,2-26,2$ & $1,14(0,1-10,9)$ & 0,90 \\
\hline História de sequestro & 1,0 & 0 & 0,0 & - & - & - \\
\hline Falecimento de pessoa próxima & 38,0 & 7 & 7,0 & $2,4-16,4$ & $1,04(0,3-2,9)$ & 0,93 \\
\hline História de abuso sexual & 1,0 & 0 & 0,0 & - & - & - \\
\hline História de furto & 6,0 & 0 & 0,0 & - & - & - \\
\hline História de agressão & 1,0 & 1 & 1,0 & $0,6-59,0$ & $2,3(0,1-39,5)$ & 0,48 \\
\hline História de assalto residencial & 1,0 & 0 & 0,0 & - & - & - \\
\hline
\end{tabular}

n=100; IC: intervalo de confiança; OR: odds ratio

Tabela 4. Ausência de dimensões de apoio social e associação com depressão gestacional em 100 mulheres atendidas em uma unidade básica de saúde na cidade de Nova Iguaçu (RJ), com intervalo de confiança de 95\%

\begin{tabular}{|c|c|c|c|c|c|c|}
\hline \multirow{2}{*}{ Apoio social } & \multicolumn{4}{|c|}{ Depressão } & \multirow{2}{*}{ OR (IC95\%) } & \multirow{2}{*}{ Valor $\mathrm{p}$} \\
\hline & $\%$ & $\mathbf{n}$ & $\%$ & IC95\% & & \\
\hline Ausência de apoio material & 33 & 6 & 6,0 & $4,1-16,0$ & $1,01(0,3-3,0)$ & 0,97 \\
\hline Ausência de apoio afetivo & 26 & 6 & 6,0 & $5,6-17,6$ & $1,55(0,5-4,6)$ & 0,43 \\
\hline Ausência de apoio emocional & 30 & 7 & 7,0 & $3,6-17,4$ & $1,53(0,5-4,4)$ & 0,36 \\
\hline Ausência de apoio de informação & 42 & 11 & 11,0 & $2,1-20,0$ & $2,58(0,9-7,3)$ & 0,07 \\
\hline Ausência de apoio de interação & 24 & 7 & 7,0 & $5,0-13,1$ & $2,43(0,8-7,2)$ & 0,10 \\
\hline
\end{tabular}

$\mathrm{n}=100$; IC: intervalo de confiança; OR: odds ratio

\section{Análise multivariada}

As variáveis associadas à depressão gestacional na análise bivariada $(\mathrm{p} \leq 0,10)$, tais como estado civil, profissão, história anterior de depressão, tabagismo, uso de álcool, história de separação/divórcio e ausência de apoio de informação e de interação positiva, entraram no modelo de regressão logística.

Os fatores que permaneceram associados significativamente à depressão na gravidez foram: estado civil (solteira), profissão (desempregada), história anterior de depressão e tabagismo, e história de separação/divórcio. Baseando-se na relevância clínica e no valor p borderline (entre 0,05 e $0,10)^{44}$, a variável "história de separação/divórcio", embora marginalmente associada à depressão gestacional $(\mathrm{p}=0,07)$, foi considerada significativa (Tabela 5).
Tabela 5. Análise multivariada dos fatores associados à depressão gestacional em 100 mulheres atendidas em uma unidade básica de saúde na cidade de Nova Iguaçu (RJ), com intervalo de confiança de 95\%

\begin{tabular}{lccc} 
& OR & IC95\% & Valor p \\
$\begin{array}{l}\text { Estado civil } \\
\quad \text { Solteira } \\
\quad \text { Casada }\end{array}$ & 6,4 & $1,5-27,0$ & 0,01 \\
$\quad$ Profissão & 1,0 & & \\
$\quad$ Desempregada & 5,5 & $1,3-21,8$ & 0,01 \\
$\quad$ Trabalho formal & 0,7 & $0,0-5,7$ & 0,71 \\
$\quad$ Trabalho informal & 0,2 & $0,1-4,3$ & 0,32 \\
$\quad$ Outra* & 1 & & \\
História anterior de depressão & 6,8 & $1,7-26,1$ & 0,01 \\
Tabagismo & 12,4 & $1,7-87,2$ & 0,01 \\
História de separação/divórcio & 3,3 & $0,8-12,6$ & 0,07 \\
\hline *Dona de casa (16\%) e estudante (11\%); $\mathrm{n}=100$; IC: intervalo de confiança;
\end{tabular}

OR: odds ratio. 


\section{DISCUSSÃO}

Este estudo estimou a prevalência da depressão gestacional e a associação com o apoio social e outros fatores de risco em mulheres durante o terceiro trimestre de gestação, atendidas no serviço de pré-natal de uma unidade básica de saúde de um município da Baixada Fluminense, Rio de Janeiro. A depressão foi diagnosticada em aproximadamente um quinto destas mulheres (18\%; IC95\% 12,2-23,8). Ser solteira, estar desempregada, possuir história anterior de depressão, tabagismo e ter história de separação/divórcio nos últimos 12 meses foram itens associados ao aumento de risco para depressão durante a gestação.

A taxa de prevalência de depressão durante a gestação neste estudo foi semelhante às relatadas em pesquisas realizadas em países desenvolvidos (10 a 15\%) e países em desenvolvimento, inclusive no Brasil, os quais relataram a prevalência de depressão gestacional entre 20 e $25 \% \%^{43,45,46}$.

Um estudo brasileiro relatou que a prevalência de depressão gestacional $(37,8 \%)$ foi notavelmente maior do que as nossas estimativas, mas a amostra era pequena $(n=33)$ e a depressão foi apurada por um questionário de rastreamento de sintomas depressivos ${ }^{28}$. Outros estudos em países desenvolvidos, que também utilizaram escalas para rastreamento de sintomas, relataram prevalência acima de $20 \%^{46-50}$. É importante enfatizar que o uso de instrumento de rastreamento tende a classificar muitos indivíduos falso-positivos como portadores de transtorno mental, aumentando a prevalência destes transtornos na população em geral. No entanto, nós utilizamos o CIDI, uma entrevista diagnóstica estruturada, padronizada, com validade para uso no Brasil e que fornece diagnóstico de depressão.

Contrariamente, alguns países desenvolvidos apresentaram prevalências de transtorno depressivo muito baixas em relação à encontrada no presente estudo. Uma pesquisa no Japão relatou prevalência de $5,6 \%$ de depressão ${ }^{14} ; 6,9 \%$ na Suécia ${ }^{23} ; 7,7 \%$ na Finlândia e $9 \%$ nos Estados Unidos ${ }^{51}$. A menor prevalência nestes países pode ser resultado de alta qualidade de assistência pré-natal, alto nível socioeconômico e menos eventos de vida estressantes, tais como dificuldades financeiras ${ }^{24,52}$

No nosso estudo, a depressão gestacional esteve associada significativamente a alguns fatores. Possuir história anterior de depressão à gestação, por exemplo, foi relacionado significativamente à depressão durante a gestação, resultado consistente com muitos outros estudos ${ }^{18,19,31,43,46,47,51,53}$.

A depressão frequentemente é uma condição crônica e recorrente, logo, podemos conjecturar que história anterior de depressão seja um fator de risco significativo para a depressão durante a gravidez $\mathrm{z}^{41,54,55}$.
A cronicidade se evidencia pela alta prevalência de depressão por toda a vida encontrada em nosso estudo (24\%). Apesar disso, ressalta-se que apenas 6\% das mulheres relataram tratamento psiquiátrico anterior, o que revela um sério e importante problema na assistência em saúde mental. Este achado é compatível com outros estudos realizados em países em desenvolvimento, nos quais uma pequena proporção de indivíduos com transtornos psiquiátricos recebem tratamento ${ }^{19,56}$.

Associações entre eventos estressantes da vida e depressão durante a gravidez também têm sido relatadas por outros estudos ${ }^{15,16,18,57,58}$. Em nossa pesquisa, a perda de um relacionamento conjugal no último ano esteve associada à depressão durante a gestação. A maioria dos estudos epidemiológicos mostra associação entre este tipo de evento e depressão, já que ocorrem mudanças identificáveis no padrão de vida que significam uma ruptura do comportamento usual e afetam o bem-estar do indivíduo ${ }^{59-61}$.

Foi possível identificar a associação entre ser solteira e depressão durante a gestação, sendo a mesma relação encontrada em outros estudos ${ }^{14,20,62,63}$, o que leva a acreditar que as pessoas que vivem sozinhas parecem ter maior risco para depressão, ou vice-versa ${ }^{64}$. Da mesma forma como estar desempregada, resultado também compatível com outras pesquisas ${ }^{19,27,41}$. De fato, alguns autores também afirmam que indivíduos desempregados, além de sofrerem de depressão, podem apresentar instabilidade emocional (distress) e perda de autoconfiança ${ }^{64,65}$. Observamos que um conjunto de situações, como o fato de ser solteira, estar desempregada e ter vivenciado recentemente uma separação conjugal, pode ter efeito aditivo para o risco de depressão durante a gestação.

As taxas de tabagismo, álcool e uso de drogas ilícitas encontradas em nosso estudo foram muito baixas em comparação a outras pesquisas. É possível que as participantes relutassem em divulgar esses comportamentos para a entrevistadora. Ainda assim, no presente trabalho o tabagismo foi associado à depressão, como apresentado por outros autores ${ }^{14,22,46}$. Há evidências de que o uso de nicotina interfere nos sistemas neuroquímicos, afetando circuitos neurais, tais como mecanismos reforçadores associados à regulação de humor ${ }^{66,67}$. Por outro lado, o indivíduo deprimido usaria o cigarro para auxiliar na "automedicação" de sentimentos de tristeza ou humor negativo ${ }^{67,68}$.

O tabagismo, além de afetar a saúde da mãe, pode gerar danos ao feto e recém-nascido, como aumento do risco de aborto, prematuridade e baixo peso ao nascimento ${ }^{69,70}$. Apesar de não ter sido significativa na análise final, é comum a associação entre transtornos psiquiátricos e consumo de álcool durante a gestação ${ }^{30,71}$. A presença de transtornos 
mentais, baixa renda, desemprego e falta de estabilidade na união civil pode colaborar para o uso de substâncias psicoativas, e vice-versa, em gestantes ${ }^{30,72}$.

Há de se ressaltar que a associação inicial de ausência de apoio de informação e interação positiva com depressão não foi confirmada pela análise final. Tal resultado é aparentemente contraditório, uma vez que a grande maioria dos estudos na área já comprovou relação entre ambos ${ }^{14,38}$. Entretanto, ao prosseguir a análise com outras variáveis, essa associação foi perdendo a significância até desaparecer. O resultado também é corroborado por outros estudos ${ }^{73-76}$.

Uma possível explicação para a falta de associação em nosso estudo é a dicotomização da pontuação da escala de apoio social, o que pode ter limitado a sua sensibilidade ${ }^{16}$. Esta caracterização em "ter ou não ter" é muito redutora e limitativa, com possíveis consequências, tais como perda de informação contida nos dados e inferências menos adequadas ${ }^{77-79}$. O tamanho reduzido da população $(n=100)$ é outro fator que pode ter influenciado na falta de associação entre ausência de apoio social e depressão na análise final. Além disso, dentro de um modelo de eventos de vida estressantes, o apoio social tem efeito amortecedor para eventos que possam gerar estresse no dia a dia. Para trabalhar neste modelo, outras variáveis devem ser incorporadas, entre elas estratégias de coping, resiliência e traço de personalidade. Esses fatores, quando analisados em conjunto, têm efeito significativo associado a transtornos psiquiátricos como a depressão ${ }^{80,81}$. Porém, isso implica em estudos mais custosos, mais instrumentos e preparo de pesquisadores, e demandam mais tempo de entrevista.

É provável que a variável "apoio social" tenha sido absorvida e incorporada a outras que também representam diminuição de apoio social devido à redução do grupo social, tais como estar solteira e desempregada, resultando em seu desaparecimento na análise final.

Embora neste estudo o apoio social não tenha permanecido associado, de maneira independente, à depressão no modelo de regressão logística, destaca-se ausência de apoio de informação e de interação, que, na análise bivariada, obteve valor p significante e borderline (entre 0,05 e 0,10 ).

A ausência de apoio de informação tem sido associada ao aparecimento de episódios depressivos durante o período de gestação $0^{82}$, o que pode ter tido contribuição da alta frequência de gravidez não planejada, portanto, da falta de informações acerca deste período de vida tão importante para a mulher. Além disso, a falta de interação com pessoas que proporcionem momentos de entretenimento leva à diminuição de sua rede social e ao isolamento, aumentando a chance de depressão ou, ainda, piorando um quadro depressivo já existente ${ }^{82}$.
Um grande número de mulheres relatou ausência de apoio material. Isto se torna preocupante por se tratar de pessoas, na sua maioria, solteiras, desempregadas e com gravidez não planejada, ou seja, uma população com características suscetíveis a transtornos mentais como a depressã $0^{53,83}$. Este fato torna-se mais um agravante em países como o Brasil, onde a taxa de desemprego é alta, principalmente entre mulheres jovens e de classe social mais baixa.

Vivenciar a gravidez sob estes fatores pode exacerbar ou desencadear a depressão, influenciando negativamente a gestação, já que os sintomas da doença interferem no desempenho das gestantes quanto ao autocuidado e adesão ao acompanhamento pré-natal.

Deste modo, sugere-se que o cuidado a essas mulheres seja oferecido por uma equipe multidisciplinar para tornar este período o menos estressante possível. As gestantes necessitam receber continuamente apoio de informação por meio de conhecimentos que possam orientar e auxiliar na tomada de decisões que beneficiem sua saúde e a de seu bebê. Já o apoio material pode ser fornecido, por pensões pagas pelo governo federal, para cobrir despesas adicionais do período de gravidez ou dela decorrentes, sob a orientação da assistência social ${ }^{20}$.

Por se tratar de um estudo seccional, não foi possível inferir causalidade entre apoio social, os demais fatores de risco e depressão devido a problemas metodológicos, como o viés temporal, visto que é difícil separar causa e efeito. Ainda assim, foi viável testar a existência de associações estatísticas entre os eventos examinados na amostra.

Os indicadores econômicos aqui apresentados sugerem que nossa população de gestantes é semelhante à de mulheres que procuram atendimento em serviços de pré-natal na rede pública do SUS ${ }^{42,43,76}$. Como este sistema atende predominantemente aos brasileiros de baixa renda, os resultados relatados aqui não devem ser generalizados para a população brasileira em geral.

\section{CONCLUSÃO}

A depressão é um transtorno comum durante a gestação e estar solteira, desempregada, possuir história anterior de depressão e história de separação/divórcio no último ano foram associados a ela durante este período. O impacto desses fatores pode ser exacerbado durante a gravidez, especialmente em países em desenvolvimento, onde as mulheres enfrentam desigualdades socioeconômicas ${ }^{41}$.

$\mathrm{O}$ uso de estudos de seguimento, com amostras maiores e significativas, que possam incluir avaliação de variáveis que 
se relacionam com apoio social e depressão, entre elas resiliência e estratégias de coping, é necessário para a aplicação em pesquisas futuras. Destaca-se que, devido à sua peculiaridade, estudos qualitativos são também importantes para ajudar na melhor compreensão do tema.

A ausência de apoio de informação e interação durante o período pré-natal e sua associação inicial com a depressão evidenciam a fragilidade do serviço de saúde no atendimento integral às gestantes. Apesar dos recursos limitados de atenção básica, o SUS preconiza as unidades básicas como referencial para o primeiro atendimento de depressão ${ }^{38}$.

As implicações deste estudo para a prática enfatizam a necessidade de triagem para depressão durante a gestação em locais onde o tratamento está disponível. A presença de uma equipe de saúde mental nos serviços de pré-natal pode auxiliar na triagem e no acompanhamento das gestantes deprimidas.

\section{REFERÊNCIAS}

1. Langford CP, Bowsher J, Maloney JP, Lillis PP. Social support: a conceptual analysis. J Adv Nurs. 1997;25(1):95-100.

2. Barrón A. Apoyo social. Aspectos teóricos y aplicaciones. Madrid: Siglo Veinteuno, 1996.

3. Galanter M. Research on social supports and mental illness. Am J Psychiatry. 1988;145(10):1270-2

4. Olstad R, Sexton H, Sogaard AJ. The Finnmark Study. A prospective population study of the social support buffer hypothesis, specific stressors and mental distress. Soc Psychiatry Psychiatr Epidemiol. 2001;36(12):582-9.

5. Mitsuhiro SS, Chalem E, Barros MM, Guinsburg R, Laranjeira R. Teenage pregnancy: use of drugs in the third trimester and prevalence of psychiatric disorders. Rev Bras Psiquiatr. 2006:28(2):122-5.

6 Pierce GR, Sarason IG, Sarason BR, Joseph HJ, Henderson CA. Conceptualizing and assessing social support in the context of the family. In: Pierce GR, Sarason BR, Sarason IG, editors. Handbook of social support and the family. New York: Plenum Press; 1996. p. 3-23.

7. Wallston BS, Alagna SW, Devellis BM, Devellis RF. Social support and health. Health Psychology. 1983;2:367-91.

8. Geller PA. Pregnancy as a stressful life event. CNS Spectr. 2004; 9(3): 188-97.

9. Matsukura TS, Marturano EM, Oishi J. O questionário de suporte social (SSQ): estudos de adaptação para o português. Rev Latino-Am Enfermagem. 2002;10(5):675-81.

10. Dalgard OS, Haheim LL. Psychosocial risk factors and mortality: a prospective study with special focus on social support, social participation, and locus of control in Norway. J Epidemiol Community Health. 1998;52(8):476-81.

11. Camacho RS, Cantinelli FS, Ribeiro CS, Cantilino A, Gonsales BK, Braguittoni E,Rennó Jr J. Transtornos psiquiátricos na gestação e no puerpério: classificação, diagnóstico e tratamento. Rev Psiquiatr Clín. 2006;33(2):92-102.

12. Bennett HA, Einarson A, Taddio A, Koren G, Einarson TR. Prevalence of depression during pregnancy: sistematic review. Obstet Gynecol. 2004;103(4):698-709.

13. Faisal-Cury A, Menezes PR. Prevalence of anxiety and depression during pregnancy in a private setting sample. Arch Womens Ment Health. 2007;10(1):25-32.

14. Kitamura T, Yoshida K, Okano T, Kinoshita K, Hayashi M, Toyoda N, Ito M, Kudo N, Tada K, Kanazawa K, Sakumoto K, Satoh S, Furukawa T, Nakano H. Multicentre prospective study of perinatal depression in
Japan: incidence and correlates of antenatal and postnatal depression. Arch Womens Ment Health. 2006;9(3):121-30.

15. Rubertsson C, Wickberg B, Gustavsson P, Radestad I. Depressive symptoms in early pregnancy, two months and one year portpartumprevalence and psychosocial risk factors in a national Swedish sample. Arch Womens Ment Health. 2005;8(2):97-104.

16. Lovisi GM, López JR, Coutinho ES, Patel V. Poverty, violence and depression during pregnancy: a survey of mothers attending a public hospital in Brazil. Psychol Med. 2005;35(10):1485-92.

17. Heron J, O'Connor TG, Evans J, Golding J, Glover V, ALSPAC Study Team. The course of anxiety and depression trough pregnancy and the postpartum in a community sample. J Affect Disord. 2004;80(1):65-73.

18. Felice E, Saliba J, Grech V, Cox J. Prevalence rates and psychosocial characteristics associated with depression in pregnancy and postpartum in Maltese women. J Affect Disord. 2004;82(2):297-301.

19. Patel V, Rodrigues M, DeSouza N. Gender, poverty, and pPostnatal depression: a study of mothers in Goa, India. Am J Psychiatry. 2002;159(1): 43-7.

20. Freitas GVS, Botega NJ. Gravidez na adolescência: prevalência de depressão, ansiedade e ideação suicida. Rev Assoc Med Bras. 2002;48(3):245-9.

21. Moreira MC, Sarriera JC. Satisfação e composição da rede de apoio social a gestantes adolescentes. Psicol Estud. 2008;13(4):781-9.

22. Elsenbruch S, Benson S, Rücke M, Rose M, Dudenhausen J, PincusKnackstedt MK, Klapp BF, Arck PC. Social support during pregnancy: effects on maternal depressive symptoms, smoking and pregnancy outcome. Hum Reprod. 2007;22(3):869-77.

23. Andersson L, Sundström-Poromaa I, Wulff M, Aström M, Bixo M. Depression and anxiety during pregnancy and six months postpartum: a follow-up study. Acta Obstet Gynecol Scand. 2006;85(8):937-44.

24. Patel V, DeSouza N, Rodrigues M. Postnatal depression and infant growth and development in low income countries: a cohort study from Goa, India. Arch Dis Child. 2003;88(1):34-7.

25. Josefsson A, Berg G, Nordin C, Sydsjö G. Prevalence of depressive symptoms in late pregnancy and postpartum. Acta Obstet Gynecol Scand. 2001;80(3):251-5.

26. Caputo VG, Bordin IA. Problemas de saúde mental entre jovens grávidas e não-grávidas. Rev Saúde Pública. 2007;41(4):573-81.

27. Mitsuhiro SS, Chalem E, Barros MM, Guinsburg R, Laranjeira R. Teenage pregnancy: use of drugs in the third trimester and prevalence of psychiatric disorders. Rev Bras Psiquiatr. 2006;28(2):122-5. 
28. Da-Silva VA, Moraes-Santos AR, Carvalho MS, Martins ML, Teixeira NA. Prenatal and postnatal depression among low income Brazilian women. Braz J Med Biol Res. 1998;31(6):799-804.

29. Falcone VM, Mäder CVN, Nascimento CFL, Santos JMM, Nóbrega FJ. Atuação multiprofissional e a saúde mental de gestantes. Rev Saúde Pública. 2005;39(4):612-8.

30. Pinheiro SN, Laprega MR, Furtado EF. Morbidade psiquiátrica e uso de álcool em gestantes usuárias do Sistema Único de Saúde. Rev Saúde Pública. 2005;39(4):593-8.

31. Luis MAV, Oliveira ER. Transtornos mentais na gravidez, parto e puerpério, na região de Ribeirão Preto-SP-Brasil. Rev Esc Enf USP. 1998,32(4):314-24.

32. Organização Mundial de Saúde. Classificação de transtornos mentais e de comportamento da CID-10. Porto Alegre: Artes Médicas, 1993.

33. American Psychiatric Association. Manual diagnóstico e estatístico de transtornos mentais. 4. ed. Porto Alegre: Artmed, 2002.

34. Quintana MI, Gastal FL, Jorge MR, Miranda CT, Anderoli SB. Validity and limitations of the Brazilian version of the Composite International Diagnostic Interview (CIDI 2.1). Rev Bras Psiquiatr. 2007;29(1):18-22.

35. Sherbourne CD, Stewart AL. The MOS social support survey. Social Science and Medicine. 1991;32:705-14.

36. Robaina JR. Queixas de insônia e menopausa entre funcionárias de uma universidade no Rio de Janeiro: estudo pró-saúde [tese]. Rio de Janeiro (RJ): Universidade do Estado do Rio de Janeiro. Instituto de Medicina Social; 2009.

37. Andrade CR, Chor D, Faerstein E, Griep RH, Lopes CS, Werneck G. Apoio social e auto-exame das mamas no Estudo Pró- Saúde. Cad Saúde Pública. 2005;21(2):379-86.

38. Chor D, Griep RH, Lopes CS, Faerstein E. Medidas de rede e apoio social no Estudo Pró-Saúde: pré-testes e estudo piloto. Cad Saúde Pública. 2001;17(4):887-96.

39. Silva KS, Coutinho ESF. Escala de apoio social aplicada a uma população de gestantes: confiabilidade teste-reteste e estrutura de concordância dos itens. Cad Saúde Pública. 2005;21(3):979-83.

40. Holmes TH, Rahe RK. The social readjustment rating scale. J Psychosom Res. 1967;11(2):213-8.

41. Lopes CS, Faerstein E, Chor D. Eventos de vida produtores de estresse e transtornos mentais comuns: resultados do Estudo Pró-Saúde. Cad Saúde Pública. 2003;19(6):1713-20.

42. Pereira PK, Lovisi GM, Lima LA, Legay LF. Complicações obstétricas, eventos estressantes, violência e depressão durante a gravidez em adolescentes atendidas em unidade básica de saúde. Rev Psiq Clín. 2010;37(5):216-22.

43. Pereira PK, Lovisi GM, Pilowsky DL, Lima LA, Legay LF. Depression during pregnancy: prevalence and risk factors among women attending a public health clinic in Rio de Janeiro, Brazil. Cad Saúde Pública. 2009;25(12):2725-36.

44. Spoozak L, Gotman N, Smith MV, Belanger K, Yonkers KA. Evaluation of a social support measure that may indicate risk of depression during pregnancy. J Affect Disord. 2009;114(1-3):216-23.

45. Blaney NT, Fernandez MI, Ethier KA, Wilson TE, Walter E, Koenig LJ. Psychosocial and behavioral correlates of depression among HIVinfected pregnant women. Aids Patient Care STDS. 2004;18(7):405-15.
46. Bowen A, Muhajarine N. Prevalence of antenatal depression in women enrolled in an outreach program in Canada. J Obstet Gynecol Neonatal Nurs. 2006;35(4):491-8.

47. Marcus SM, Flynn HA, Blow FC, Barry KL. Depressive symptoms among pregnant women screened in obstetrics settings. J Womens Health. 2003;12(4):373-80.

48. Kim HG, Mandell M, Crandall C, Kuskowski MA, Dieperink B, Buchberger RL. Antenatal psychiatric illness and adequacy of prenatal care in an ethnically diverse inner-city obstetric population. Arch Womens Ment Health. 2006;9(2):103-7.

49. Adouard F, Glangeaud-Freudenthal NM, Golse B. Validation of the Edinburgh postnatal depression scale (EPDS) in a sample of women with high-risk pregnancies in France. Arch Womens Ment Health. 2005;8(2):89-95.

50. Olstad R, Sexton H, Sogaard AJ. The Finnmark Study. A prospective population study of the social support buffer hypothesis, specific stressors and mental distress. Soc Psychiatry Psychiatr Epidemiol. 2001;36(12):582-9.

51. Kurki T, Hiilesmaa V, Raitasalo R, Mattila H, Ylinkorkala O. Depression and anxiety in early pregnancy and risk for preeclampsia. Obstet Gynecol. 2000;95(4):487-90.

52. Rich-Edwards JW, Kleinman K, Abrams A, Harlow BL, McLaughlin TJ, Joffe H, Gillman MW. Sociodemographic predictors of antenatal and postpartum depressive symptoms among women in a medical group practice. J Epidemiol Community Health. 2006;60(3):221-7.

53. Lee DT, Kleinman J, Kleinman A. Rethinking depression: an ethnographic study of the experiences of depression among Chinese. Harv Rev Psychiatry. 2007;15(1):1-8.

54. Chee CY, Lee DT, Chong YS, Tan LK, Ng TP, Fones CS. Confinement and other psychosocial factors in perinatal depression: a transcultural study in Singapore. J Affect Disord. 2005;89(1-3):157-66.

55. Cohen LS, Altshuler LL, Harlow BL, Nonacs R, Newport DJ, Viguera AC, Suri R, Burt VK, Hendrick V, Reminick AM, Loughead A, Vitonis AF, Stowe ZN. Relapse of major depression during pregnancy in women who maintain or discontinue antidepressant treatment. JAMA. 2006;295(5):499-507.

56. Herring M, Kaslow NJ. Depression and attachment in families: a childfocused perspective. Fam Process. 2002;41(3):494-518.

57. Theme-Filha MM, Szwarcwald CL, Souza-Júnior PR. Socio-demographic characteristics, treatment coverage, and self-rated health of individuals who reported six chronic diseases in Brazil, 2003. Cad Saude Publica. 2005;21(Suppl: 43-53).

58. Gulseren L, Erol A, Gulseren S, Kuey L, Kilic B, Ergor G. From antepartum to postpartum: a prospective study on the prevalence of peripartum depression in a semiurban Turkish community. J Reprod Med. 2006;51(12):955-60.

59. Fiscella K. Is lower income associated with greater biopsychosocial morbidity? Implications for physicians working with underserved patients. J Fam Pract. 1999;48(5):372-7.

60. Gordon HS, Rosenthal GE. Impact of marital status on outcomes in hospitalized patients. Evidence from an academic medical center. Arch Intern Med. 1995;155(22):2465-71.

61. Kessing LV, Agerbo E, Mortensen PB. Does the impact of major stressful life events on the risk of developing depression change throughout life? Psychol Med. 2003;33(7):1177-84. 
62. Booth A, Amato P. Divorce and psychological stress. J Health Soc Behav. 1991;32(4):396-407.

63. Mckee MD, Cunningham M, Jankowski KR, Zayas L. Health-related functional status in pregnancy: relationship to depression and social support in a multi-ethnic population. Obstet Gynecol. 2001;97(6):988-93.

64. Webster J, Linnane JW, Dibley LM, Hinson JK, Starrenburg SE, Roberts JA. Measuring social support in pregnancy: can it be simple and meaningful? Birth. 2000;27(2):97-101.

65. Bahls SC. Depressão: uma breve revisão dos fundamentos biológicos e cognitivos. Interação. 1999;3:49-60.

66. Morrell S, Taylor R, Quine S, Kerr C, Western J. A cohort study of unemployment as a cause of psychological disturbance in Australian youth. Soc Sci Med. 1994;38(11):1553-64.

67. Bhugra D. Unemployment, poverty and homelessness. In: Bhugra D, Leff J, editors. Principles of social psychiatry. Oxford: Blackwell Science; 1997. p. 355-84.

68. Windle M, Windle RC. Depressive symptoms and cigarette smoking among middle adolescents: prospective associations and intrapersonal and interpersonal influences. J Consult Clin Psychol. 2001;69(2):215-26.

69. Anda RF, Williamson DF, Escobedo LG, Mast EE, Giovino GA, Remington PL. Depression and the dynamics of smoking. A national perspective. JAMA. 1990;264(12):1541-5

70. Breslau N, Kilbey M, Andreski P. Nicotine dependence, major depression, and anxiety in young adults. Arch Gen Psychiatry. 1991;48(12):1069-74.

71. Vik T, Jacobsen G, Vatten L, Bakketeig LS. Pre and postnatal growth in childen of women who smoked in pregnancy. Early Hum Dev. 1996;45:245-55.

72. Severson HH, Andrews JA, Lichtenstein E, Wall M, Zoref L. Predictors of smoking during and after pregnancy: a survey of mothers of newborns. Prev Med. 1995;24(1):23-8.

73. Kelly R, Zatzick D, Anders T. The detection and treatment of psychiatric disorders and substance use among pregnant women cared for in obstetrics. Am J Psychiatry. 2001;158(2):213-9.
74. Ebrahim SH, Gfroerer J. Pregnancy-related substance use in the United States during 1996-1998. Obstet Gynecol. 2003;101(2):374-9.

75. Ethier KA, Ickovics JR, Fernandez MI, Wilson TE, Royce RA, Koenig LJ. The perinatal guidelines evaluation project HIV and pregnancy study: overview and cohort description. Public Health Rep. 2002; 117(2):137-47.

76. Da Costa D, Larouche J, Dritsa M, Brender W. Psychosocial correlates of prepartum and postpartum depressed mood. J Affect Disord. 2000;59(1):31-40.

77. Lackner JB, Joseph JG, Ostrow DG, Kessler RC, Eshleman S, Wortman CB, O'Brien K, Phair JP, Chmiel J. A longitudinal study of psychological distress in a cohort of gay men. Effects of social support and coping strategies. J Nerv Ment Dis. 1993;181(1):4-12.

78. Lovisi GM, Milani I, Caetano G, Abelha L, Morgado AF. Suporte social e distúrbios psiquiátricos: em que base se alicerça a associação? Inform Psiq. 1996;15(2):65-8.

79. Abreu MN, Siqueira AL, Cardoso CS, Caiaffa WT. Ordinal logistic regression models: application in quality of life syudies. Cad Saude Publica. 2008;24(Suppl 4):S581-91.

80. Leal J, Garganta R, Seabra A, Chaves R, Souza M, Maia J. Um resumo do estado da arte acerca da Síndrome Metabólica. Conceito, operacionalização, estratégias de análise estatística e sua associação a níveis distintos de actividade física. Rev Port Cien Desp. 2009; 9(2-3):231-44.

81. Connor-Smith JK, Flachsbart C. Relations between personality and coping: a meta-analysis. J Pers Soc Psychol. 2007;93(6):1080-107.

82. Parry G, Shapiro DA. Social support and life events in working class women. Stress buffering or independent effects? Arch Gen Psychiatry. 1986;43(4):315-23.

83. Freire SA, Sommerhalder C. Envelhecer nos tempos modernos. In: Neri AL, Freire SA, editors. E por falar em boa velhice. Campinas: Papirus; 2002. p. 125-35

Recebido em: 21/05/2012 Aprovado em: 11/10/2012 